\title{
Modelling, Analyzing and Control of Interactions Among Agents in MAS ${ }^{\star}$
}

\author{
František Čapkovič \\ Institute of Informatics, Slovak Academy of Sciences \\ Dúbravská cesta 9, 84507 Bratislava, Slovak Republic \\ Frantisek.Capkovic@savba.sk, utrrcapk@savba.sk \\ http://www.ui.sav.sk/home/capkovic/capkhome.htm
}

\begin{abstract}
An alternative approach to modelling and analysis of interactions among agents in multiagent systems (MAS) and their control is presented in analytical terms. The reachability graph of the Petri net (PN)-based model of MAS is found as well as the space of feasible states. Trajectories representing the interaction processes among agents in MAS are computed by means of the mutual intersection of both the straightlined reachability tree (from a given initial state towards the terminal one) and the backtracking reachability tree (from the desired terminal state towards the initial one however, oriented towards the terminal state). Control interferences are obtained on base of the most suitable trajectory chosen from the set of feasible ones.
\end{abstract}

\section{Introduction}

MAS are used in intelligent control, especially for a cooperative problem-solving [14. To analyze complicated interactions among agents modelling of them is often used. The negotiation belongs to the most important interactions. It is the process of multilateral bargaining for mutual profit. In other words [13], [1], the negotiation is a decision process where two or more participants make individual decisions and interact with each other in order to reach a compromise. In [9] PN are used for e-negotiations activities. PN were chosen to model MAS too [12], [11. On the base of previous experience [3, 4, [7] with PN-based modelling and control synthesis of the discrete event dynamic systems (DEDS) and the agent cooperation [5], 6] a new approach to modelling, analysis and control of the negotiation process is proposed here. The negotiation process is understood to be DEDS. The approach consists of: 1 . creating the PN-based mathematical model of the negotiation process; 2 . generating the space of feasible states which are reachable from the given initial state; 3 . utilizing the reachability graph $(\mathrm{RG})$ in order to find the feasible state trajectories to a prescribed feasible terminal state. After a thorough analyzing the set of possibilities, the most suitable strategy (the control trajectory) can be chosen.

\footnotetext{
* Partially supported by the Slovak Grant Agency for Science (VEGA) under grant \# 2/6102/26.
} 


\section{The PN-Based Mathematical Model of DEDS}

The analytical model of DEDS has the form

$$
\begin{aligned}
\mathbf{x}_{k+1} & =\mathbf{x}_{k}+\mathbf{B} \cdot \mathbf{u}_{k} \quad, \quad k=0, \ldots, N \\
\mathbf{B} & =\mathbf{G}^{T}-\mathbf{F} \\
\mathbf{F} \cdot \mathbf{u}_{k} & \leq \mathbf{x}_{k}
\end{aligned}
$$

where $k$ is the discrete step of the dynamics development; $\mathbf{x}_{k}=\left(\sigma_{p_{1}}^{k}, \ldots, \sigma_{p_{n}}^{k}\right)^{T}$ is the $n$-dimensional state vector of DEDS in the step $k ; \sigma_{p_{i}}^{k} \in\left\{0,1, \ldots, c_{p_{i}}\right\}$, $i=1, \ldots, n$ express the states of the DEDS elementary subprocesses or operations by 0 (passivity) or by $0<\sigma_{p_{i}} \leq c_{p_{i}}$ (activity); $c_{p_{i}}$ is the capacity of the DEDS subprocess $p_{i}$ as to its activities; $\mathbf{u}_{k}=\left(\gamma_{t_{1}}^{k}, \ldots, \gamma_{t_{m}}^{k}\right)^{T}$ is the $m$-dimensional control vector of the system in the step $k$; its components $\gamma_{t_{j}}^{k} \in\{0,1\}, j=$ $1, \ldots, m$ represent occurring of the DEDS elementary discrete events (e.g. starting or ending the elementary subprocesses or their activities, failures, etc.) by 1 (presence of the corresponding discrete event) or by 0 (absence of the event); $\mathbf{B}, \mathbf{F}, \mathbf{G}$ are structural matrices of constant elements; $\mathbf{F}=\left\{f_{i j}\right\}_{n \times m}, f_{i j} \in$ $\left\{0, M_{f_{i j}}\right\}, i=1, \ldots, n, j=1, \ldots, m$ expresses the causal relations among the states of the DEDS (in the role of causes) and the discrete events occuring during the DEDS operation (in the role of consequences) by 0 (nonexistence of the corresponding relation) or by $M_{f_{i j}}>0$ (existence and multiplicity of the relation); $\mathbf{G}=\left\{g_{i j}\right\}_{m \times n}, g_{i j} \in\left\{0, M_{g_{i j}}\right\}, i=1, \ldots, m, j=1, \ldots, n$ expresses very analogically the causal relations among the discrete events (causes) and the DEDS states (consequences); the structural matrix $\mathbf{B}$ is given by means of the arcs incidence matrices $\mathbf{F}$ and $\mathbf{G}$ according to (2); $(.)^{T}$ symbolizes the matrix or vector transposition. PN marking was denoted here by the letter $\mathbf{x}$ usually denoting the state in system theory. Fuzzy PN [10] can be modelled analogically - see [2]. Higher-level PN models usually cannot be expressed in such a pure analytical form although they are advantageous as to the smaller dimensionality.

\subsection{The PN-Based Model of the Negotiation Process Principle}

The negotiation process itself consists of several principle activities [9]. Especially, the following ones are most important: defining the negotiation environment, initial contact of agents, offer(s) and counter offer(s) among them, evaluation of proposals, and outcomes of the negotiation process. The coordination plan of the negotiation process can be formally described by DEDS modelled by PN as it can be seen on the left in Fig. 1. The PN places represent the activities and the PN transitions represent the discrete events. The interpretation of the places and transitions is the following: $p_{1}=$ start; $p_{2}=$ define negotiation environment; $p_{3}=$ initial contact; $p_{4}=$ offer(s) and counter offer $(\mathrm{s}) ; p_{5}=$ evaluation; $p_{6}=$ outcomes; $p_{7}=$ end $t_{1}=$ starting negotiation process $; t_{2}=$ negotiation plan $(\mathrm{s}) ; t_{3}=$ "hand shake"; $t_{4}=\operatorname{proposal}(\mathrm{s}) ; t_{5}=\operatorname{revised} \operatorname{proposal}(\mathrm{s}) ; t_{6}=$ agreement or quit; $t_{7}=$ ending negotiation process. However, the reality is more complicated. To illustrate it in details, let us introduce the PN-based model of the agent in general as well as that of more agents working in frame of MAS. 

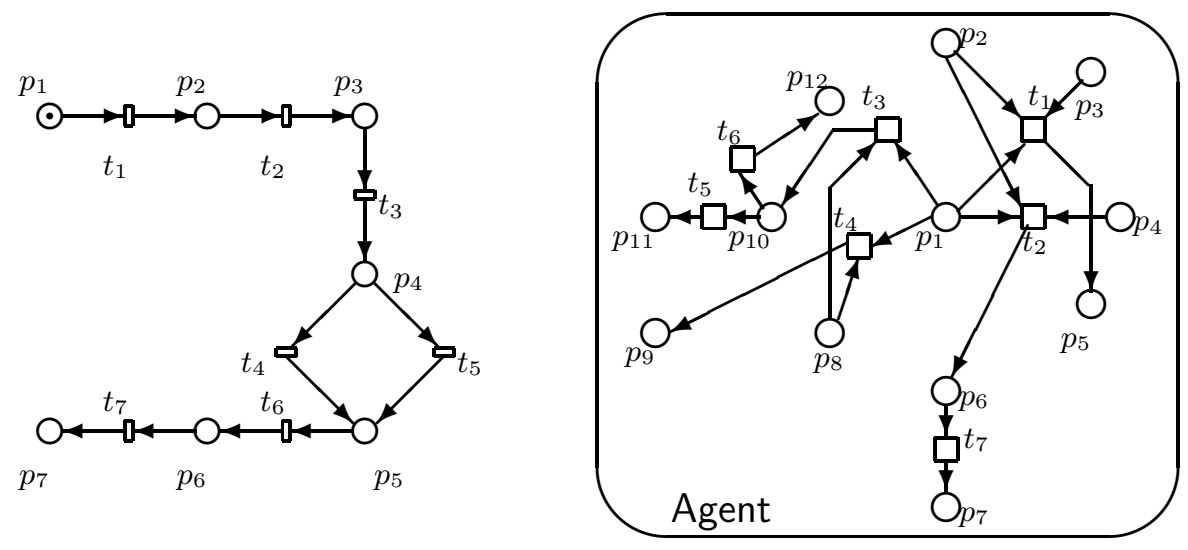

Fig. 1. The PN-based model of the coordination plan of the negotiation process in general (on the left) and the PN-based model of a general agent (on the right)

\subsection{The PN-Based Model of Agents in MAS}

The general structure of a general agent from the collaboration/negotiation point of view is given on the right in Figure 1 representing the PN-based model of the agent. To interpret the sense of the PN places and transitions, it is necessary to introduce: $p_{1}=$ the agent $\left(A_{1}\right)$ is free; $p_{2}=$ a problem has to be solved by $A_{1} ; p_{3}=A_{1}$ is able to solve the problem $\left(P_{A_{1}}\right) ; p_{4}=A_{1}$ is not able to solve $P_{A_{1}} ; p_{5}=P_{A_{1}}$ is solved; $p_{6}=P_{A_{1}}$ cannot be solved by $A_{1}$ and another agent(s) should be contacted; $p_{7}=A_{1}$ asks another agent(s) to help him to solve $P_{A_{1}}$; $p_{8}=A_{1}$ is asked by another agent(s) to solve a problem $P_{B} ; p_{9}=A_{1}$ refuses the help; $p_{10}=A_{1}$ accepts the request of another agent(s) for help; $p_{11}=A_{1}$ is not able to solve $P_{B} ; p_{12}=A_{1}$ is able to solve $P_{B}$. In such a case parameters of the PN-based model are the following

$$
\mathbf{F}^{T}=\left(\begin{array}{llllllllllll}
1 & 1 & 1 & 0 & 0 & 0 & 0 & 0 & 0 & 0 & 0 & 0 \\
1 & 1 & 0 & 1 & 0 & 0 & 0 & 0 & 0 & 0 & 0 & 0 \\
1 & 0 & 0 & 0 & 0 & 0 & 0 & 1 & 0 & 0 & 0 & 0 \\
1 & 0 & 0 & 0 & 0 & 0 & 0 & 1 & 0 & 0 & 0 & 0 \\
0 & 0 & 0 & 0 & 0 & 0 & 0 & 0 & 0 & 1 & 0 & 0 \\
0 & 0 & 0 & 0 & 0 & 0 & 0 & 0 & 0 & 1 & 0 & 0 \\
0 & 0 & 0 & 0 & 0 & 1 & 0 & 0 & 0 & 0 & 0 & 0
\end{array}\right) ; \quad \mathbf{G}=\left(\begin{array}{llllllllllll}
0 & 0 & 0 & 0 & 1 & 0 & 0 & 0 & 0 & 0 & 0 & 0 \\
0 & 0 & 0 & 0 & 0 & 1 & 0 & 0 & 0 & 0 & 0 & 0 \\
0 & 0 & 0 & 0 & 0 & 0 & 0 & 0 & 0 & 1 & 0 & 0 \\
0 & 0 & 0 & 0 & 0 & 0 & 0 & 0 & 1 & 0 & 0 & 0 \\
0 & 0 & 0 & 0 & 0 & 0 & 0 & 0 & 0 & 0 & 1 & 0 \\
0 & 0 & 0 & 0 & 0 & 0 & 0 & 0 & 0 & 0 & 0 & 1 \\
0 & 0 & 0 & 0 & 0 & 0 & 1 & 0 & 0 & 0 & 0 & 0
\end{array}\right)
$$

Analyse e.g. the situation when $\mathbf{x}_{0}=(1,0,0,0,0,0,0,1,0,0,0,0)^{T}$, i.e. the situation when the agent $A_{1}$ is free and it is asked by the agent $A_{2}$ to solve the problem $P_{B}=P_{A_{2}}$. Using the algorithm introduced in [4] we have the following quasi-adjacency matrix $\mathbf{A}$ (its elements are the indices of the PN transitions) of the $\mathrm{PN}$ reachability tree and the matrix $\mathbf{X}_{\text {reach }}$ with columns beeing the feasible states (the initial state and all states reachable from this initial state) 


$$
\mathbf{A}=\left(\begin{array}{ccccc}
0 & 3 & 4 & 0 & 0 \\
0 & 0 & 0 & 5 & 6 \\
0 & 0 & 0 & 0 & 0 \\
0 & 0 & 0 & 0 & 0 \\
0 & 0 & 0 & 0 & 0
\end{array}\right) ; \quad \mathbf{X}_{\text {reach }}^{T}=\left(\begin{array}{cccccccccccc}
1 & 0 & 0 & 0 & 0 & 0 & 0 & 1 & 0 & 0 & 0 & 0 \\
0 & 0 & 0 & 0 & 0 & 0 & 0 & 0 & 0 & 1 & 0 & 0 \\
0 & 0 & 0 & 0 & 0 & 0 & 0 & 0 & 1 & 0 & 0 & 0 \\
0 & 0 & 0 & 0 & 0 & 0 & 0 & 0 & 0 & 0 & 1 & 0 \\
0 & 0 & 0 & 0 & 0 & 0 & 0 & 0 & 0 & 0 & 0 & 1
\end{array}\right)
$$

The reachability graph of such a PN-based model is given in Fig. 2, Such a

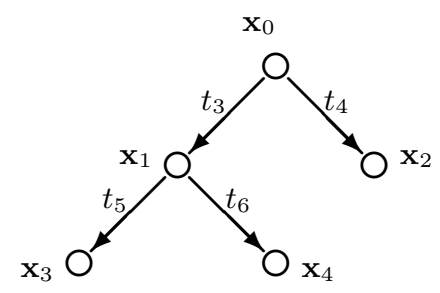

Fig. 2. The reachability graph of the agent situation

PN-based model of the agent is universal and it can be used for modelling other agents of MAS too. Namely, the same interpretation of places (however with shifted numbering $\left.p_{i+12}, i=1, \ldots, 12\right)$ can be used e.g for the agent $A_{2}$. Having two agents $A_{1}, A_{2}$ the collaboration/negotiation of them is given in Figure 3 . In case of more agents - e.g. $N_{A}$ - the places numbering of the agent $k=1, \ldots, N_{A}$ is $p_{i+12 . j}, i=1, \ldots, 12, j=k-1=0, \ldots, N_{A}-1$. In case of several agents both the PN model and the RG will be more complex. While the model size depends on modules and their interface, the RG size depends on $\mathbf{x}_{0}$ and on the structure of model blocks.

As we can see we have $n=24$ places and $m=20$ transitions in the PN model of the two agents cooperation. However, the number of transitions is higher than a simple sum being $m=14$. Namely, some transitions have to be added as an interface in order to connect both of the agents. Consequently, for two agents $A_{1}, A_{2}$ the matrices of the MAS parameters will have the form as follows, where the structure of the actual contact interface between the agents is given by the $(n \times 6)$-dimensional matrix $\mathbf{F}_{c}$ and $(6 \times n)$-dimensional matrix $\mathbf{G}_{c}$

$$
\begin{aligned}
& \mathbf{F}=\left(\begin{array}{lll}
\mathbf{F}_{1} & \mathbf{0} & \mathbf{F}_{c_{1}} \\
\mathbf{0} & \mathbf{F}_{2} & \mathbf{F}_{c_{2}}
\end{array}\right) ; \quad \mathbf{G}=\left(\begin{array}{ll}
\mathbf{G}_{1} & \mathbf{0} \\
\mathbf{0} & \mathbf{G}_{1} \\
\mathbf{G}_{c_{1}} & \mathbf{G}_{c_{2}}
\end{array}\right) \\
& \mathbf{F}_{c}^{T}=\left(\begin{array}{cccccccccccc|cccccccccccc}
0 & 0 & 0 & 0 & 0 & 0 & 0 & 0 & 0 & 0 & 0 & 0 & 0 & 0 & 0 & 0 & 0 & 0 & 1 & 0 & 0 & 0 & 0 & 0 \\
0 & 0 & 0 & 0 & 0 & 0 & 0 & 0 & 0 & 0 & 0 & 1 & 0 & 0 & 0 & 0 & 0 & 0 & 0 & 0 & 0 & 0 & 0 & 0 \\
0 & 0 & 0 & 0 & 0 & 0 & 0 & 0 & 0 & 0 & 0 & 0 & 0 & 0 & 0 & 0 & 0 & 0 & 0 & 0 & 0 & 0 & 0 & 1 \\
0 & 0 & 0 & 0 & 0 & 0 & 1 & 0 & 0 & 0 & 0 & 0 & 0 & 0 & 0 & 0 & 0 & 0 & 0 & 0 & 0 & 0 & 0 & 0 \\
0 & 0 & 0 & 0 & 0 & 0 & 0 & 0 & 0 & 0 & 0 & 0 & 0 & 0 & 0 & 0 & 0 & 0 & 0 & 0 & 0 & 1 & 0 & 0 \\
0 & 0 & 0 & 0 & 0 & 0 & 0 & 0 & 0 & 1 & 0 & 0 & 0 & 0 & 0 & 0 & 0 & 0 & 0 & 0 & 0 & 0 & 0 & 0
\end{array}\right)=\left(\mathbf{F}_{c_{1}}^{T} \mid \mathbf{F}_{c_{2}}^{T}\right)
\end{aligned}
$$




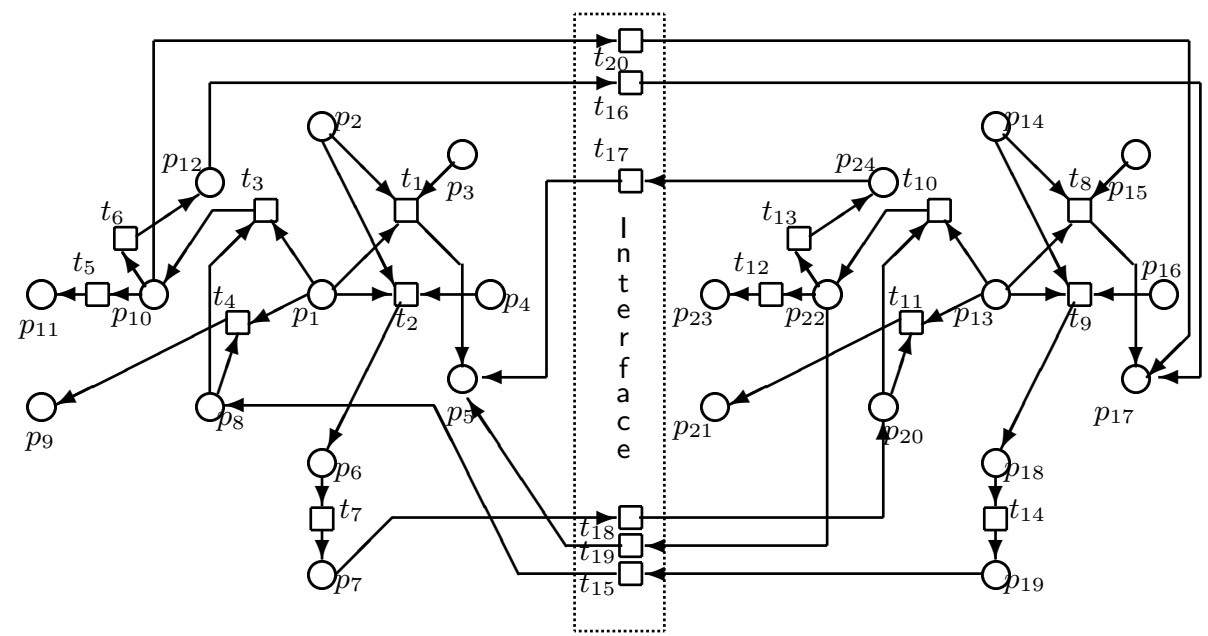

Fig. 3. The Petri net-based model of the two agents cooperation and/or negotiation by means of the interface

$$
\mathbf{G}_{c}=\left(\begin{array}{llllllllllll|llllllllllll}
0 & 0 & 0 & 0 & 0 & 0 & 0 & 1 & 0 & 0 & 0 & 0 & 0 & 0 & 0 & 0 & 0 & 0 & 0 & 0 & 0 & 0 & 0 & 0 \\
0 & 0 & 0 & 0 & 0 & 0 & 0 & 0 & 0 & 0 & 0 & 0 & 0 & 0 & 0 & 0 & 1 & 0 & 0 & 0 & 0 & 0 & 0 & 0 \\
0 & 0 & 0 & 0 & 1 & 0 & 0 & 0 & 0 & 0 & 0 & 0 & 0 & 0 & 0 & 0 & 0 & 0 & 0 & 0 & 0 & 0 & 0 & 0 \\
0 & 0 & 0 & 0 & 0 & 0 & 0 & 0 & 0 & 0 & 0 & 0 & 0 & 0 & 0 & 0 & 0 & 0 & 0 & 1 & 0 & 0 & 0 & 0 \\
0 & 0 & 0 & 0 & 1 & 0 & 0 & 0 & 0 & 0 & 0 & 0 & 0 & 0 & 0 & 0 & 0 & 0 & 0 & 0 & 0 & 0 & 0 & 0 \\
0 & 0 & 0 & 0 & 0 & 0 & 0 & 0 & 0 & 0 & 0 & 0 & 0 & 0 & 0 & 0 & 1 & 0 & 0 & 0 & 0 & 0 & 0 & 0
\end{array}\right)=\left(\mathbf{G}_{c_{1}} \mid \mathbf{G}_{c_{2}}\right)
$$

In general, for agents $i=1,2, \ldots, N_{A}$ the structure of matrices is the following

$$
\begin{aligned}
& \mathbf{F}=\left(\begin{array}{llllll}
\mathbf{F}_{1} \mathbf{0} & \ldots & \mathbf{0} & \mathbf{0} & \mid \mathbf{F}_{c_{1}} \\
\mathbf{0} & \mathbf{F}_{2} & \ldots & \mathbf{0} & \mathbf{0} & \mid \mathbf{F}_{c_{2}} \\
\vdots & \vdots & \ddots & \vdots & \vdots & \vdots \\
\mathbf{0} & \mathbf{0} & \ldots & \mathbf{F}_{N_{A}-1} & \mathbf{0} & \mathbf{F}_{c_{N_{A}-1}} \\
\mathbf{0} & \mathbf{0} & \ldots & \mathbf{0} & \mathbf{F}_{N_{A}} & \mathbf{F}_{c_{N_{A}}}
\end{array}\right) ; \mathbf{G}=\left(\begin{array}{lllll}
\mathbf{G}_{1} & \mathbf{0} & \ldots & \mathbf{0} & \mathbf{0} \\
\mathbf{0} & \mathbf{G}_{2} & \ldots & \mathbf{0} & \mathbf{0} \\
\vdots & \vdots & \ddots & \vdots & \vdots \\
\mathbf{0} & \mathbf{0} & \ldots & \mathbf{G}_{N_{A}-1} & \mathbf{0} \\
\mathbf{0} & \mathbf{0} & \ldots & \mathbf{0} & \mathbf{G}_{N_{A}} \\
\hdashline \mathbf{G}_{c_{1}} & \mathbf{G}_{c_{2}} & \ldots & \mathbf{G}_{c_{N_{A}-1}} & \mathbf{G}_{c_{N_{A}}}
\end{array}\right) \\
& \mathbf{B}=\left(\begin{array}{lllll|l}
\mathbf{B}_{1} \mathbf{0} & \ldots \mathbf{0} & \mathbf{0} & \mathbf{B}_{c_{1}} \\
\mathbf{0} & \mathbf{B}_{2} \ldots \mathbf{0} & \mathbf{0} & \mid \mathbf{B}_{c_{2}} \\
\vdots & \vdots & \ddots & \vdots & \vdots & \vdots \\
\mathbf{0} & \mathbf{0} & \ldots & \mathbf{B}_{N_{A}-1} & \mathbf{0} & \mathbf{B}_{c_{N_{A}-1}} \\
\mathbf{0} & \mathbf{0} & \ldots & \mathbf{0} & \mathbf{B}_{N_{A}} & \mathbf{B}_{c_{N_{A}}}
\end{array}\right)=\left(\operatorname{blockdiag}\left(\mathbf{B}_{i}\right)_{i=1, N_{A}} \mid \mathbf{B}_{c}\right) \\
& \mathbf{B}_{i}=\mathbf{G}_{i}^{T}-\mathbf{F}_{i} ; \mathbf{B}_{c_{i}}=\mathbf{G}_{c_{i}}^{T}-\mathbf{F}_{c_{i}} ; i=1,2 \ldots, N_{A} ; \mathbf{F}_{c}=\left(\mathbf{F}_{c_{1}}^{T}, \mathbf{F}_{c_{2}}^{T}, \ldots, \mathbf{F}_{c_{N_{A}}}^{T}\right)^{T} \\
& \mathbf{G}_{c}=\left(\mathbf{G}_{c_{1}}, \mathbf{G}_{c_{2}}, \ldots, \mathbf{G}_{c_{N_{A}}}\right) ; \mathbf{B}_{c}=\left(\mathbf{B}_{c_{1}}^{T}, \mathbf{B}_{c_{2}}^{T}, \ldots, \mathbf{B}_{c_{N_{A}}}^{T}\right)^{T}
\end{aligned}
$$


with $\mathbf{F}_{i}, \mathbf{G}_{i}, \mathbf{B}_{i}, i=1,2, \ldots, N_{A}$, representing the parameters of the PN-based model of the agent $A_{i}$, and with $\mathbf{F}_{c}, \mathbf{G}_{c}, \mathbf{B}_{c}$ representing the structure of the actual contact interface between the agents cooperating in MAS. Using the own graphical tool (developed during last years by master students) for drawing the model by means of icons the properties of the model can be tested. The reachability tree (RT) being the most important instrument on this way can be developed in the graphical form. Starting from the initial state $\mathbf{x}_{0}=\left(\sigma_{p_{1}}, \ldots, \sigma_{p_{n}}\right)^{T}=$ $(1,1,1,0,0,0,0,0,0,0,0,0,1,1,0,1,0,0,0,0,0,0,0,0)^{T}$, i.e. from the state where only six places have nonzero marking, namely $\sigma_{p_{1}}=1, \sigma_{p_{2}}=1, \sigma_{p_{3}}=1$, $\sigma_{p_{13}}=1, \sigma_{p_{14}}=1, \sigma_{p_{16}}=1$, we can draw the reachability graph of the PNbased model of two agents cooperation given in Figure 4. It has $N=13$ nodes representing the feasible states $\left\{\mathbf{x}_{0}, \mathbf{x}_{1}, \ldots, \mathbf{x}_{12}\right\}$ of the system state space.

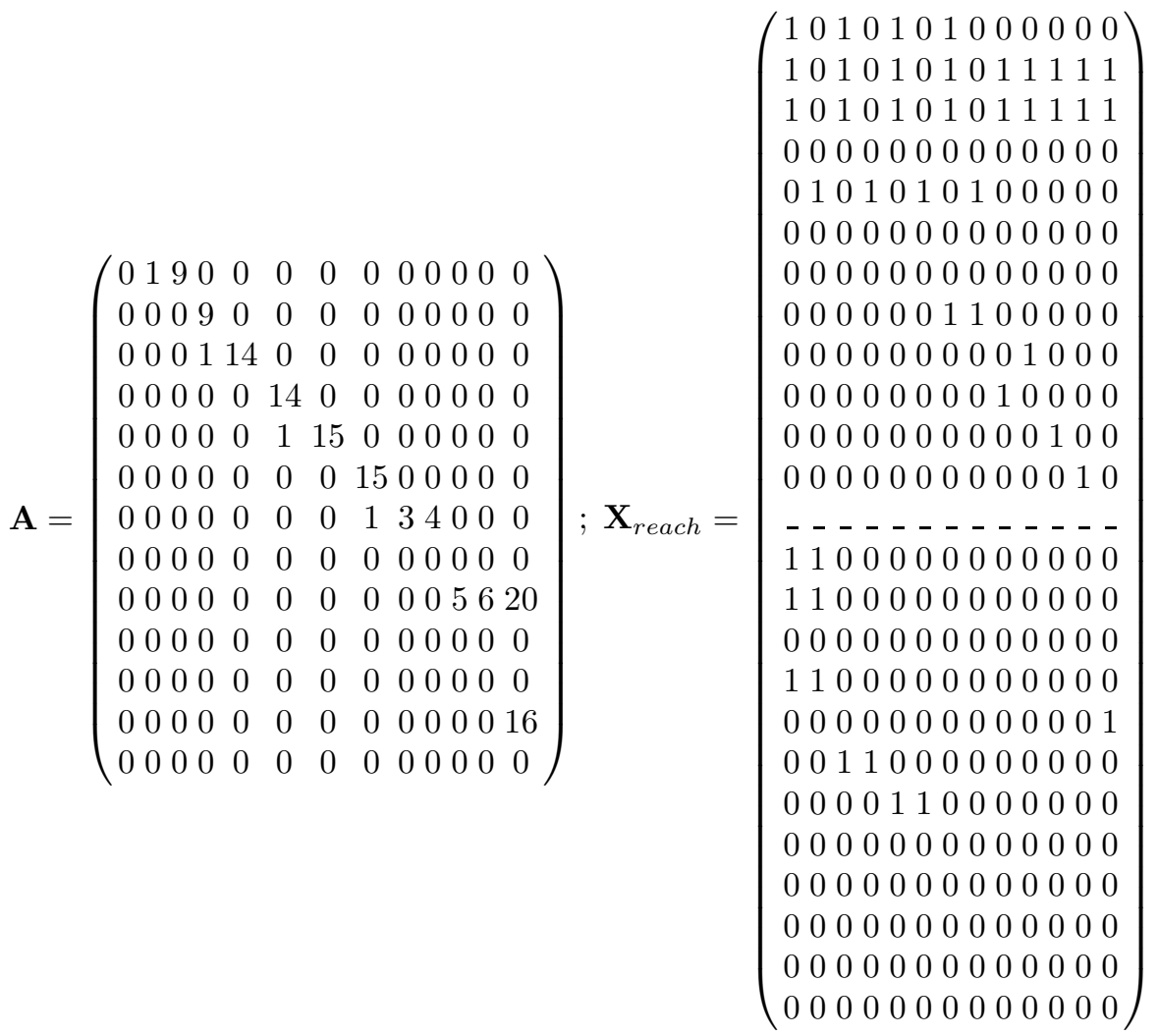

The edges express feasible paths (trajectories) among the corresponding states. Such an approach to analysing DEDS is very comfortable and user friendly. However, from the ergonomy point of view, the observed area as well as the number of variables which the human operator is able to recognize are limited. Hence, more formal approaches are found for DEDS analysis and control. Thus, large-scale mathematical models can be handled too (e.g. by means of Matlab). 


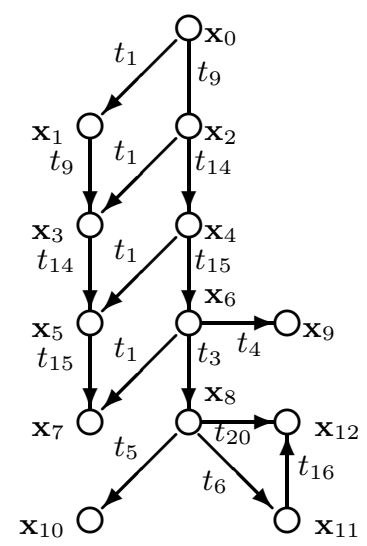

Fig. 4. The reachability graph of the two agents cooperation

\section{Analyzing the Model and the Control Synthesis}

In 8 the Matlab procedure enumerating the $\left(n_{R T} \times n_{R T}\right)$-dimensional quasifunctional adjacency matrix $\mathbf{A}$ of the RG and the space of the reachable states in the form of the $\left(n \times n_{R T}\right)$-dimensional matrix $\mathbf{X}_{\text {reach }}$ was presented. The matrices fully characterize the RG. The $\mathbf{X}_{\text {reach }}$ columns (the RG nodes) are the PN state vectors $\mathbf{x}_{0}, \mathbf{x}_{1}, \mathbf{x}_{2}, \ldots, \mathbf{x}_{n_{R T}-1}$ reachable from the initial state $\mathbf{x}_{0}$. The inputs of the procedure are the matrices $\mathbf{F}, \mathbf{G}^{T}$ and the initial state vector $\mathbf{x}_{0}$. The DEDS control synthesis based on the simple idea was also presented in [8]: (i) developing the straight-lined reachability tree (SLRT) from the given initial state $\mathbf{x}_{0}$ towards the prescribed terminal state $\mathbf{x}_{t}$; (ii) developing the backtracking reachability tree (BTRT) from $\mathbf{x}_{t}$ towards $\mathbf{x}_{0}$ however, directed to $\mathbf{x}_{t}$; (iii) intersecting both the SLRT and BTRT. All of the steps are performed numerically in Matlab. To compute SLRT the graph-based model $\mathbf{X}(k+1)=\mathbf{A}_{k}^{T} \cdot \mathbf{X}(k), k=0,1, \ldots, N-1$ is used while for computing BTRT the model $\mathbf{X}(k-1)=\mathbf{A}_{k-1} \cdot \mathbf{X}(k), k=N, \ldots 1$, with $N \leq n_{R T}-1$, is used. $\mathbf{A}_{k}$ is the functional ( $k$-variant) adjacency matrix, $\mathbf{X}(k), k=0,1, \ldots, n_{R T}-1$ are the hyperstate vectors - the unit vectors with only one nonzero element equal to 1 on the position corresponding to the index of the $\mathbf{X}_{\text {reach }}$ column. The hyperstate vector $\mathbf{X}(0)$ corresponding to $\mathbf{x}_{0}$ has 1 in its first position while the hyperstate vector corresponding to the terminal state $\mathbf{x}_{t}$ appearing e.g. in the column $j$ of $\mathbf{X}_{\text {reach }}$ will have 1 in the position $j$ i.e. it will be the hyperstate vector $\mathbf{X}(j)$. After intersection of both SLRT and BTRT the feasible trajectory (or several ones) starting from $\mathbf{x}_{0}$ and finishing in $\mathbf{x}_{t}$ is (are) obtained. Having the set of feasible trajectories, the most suitable one (satisfying imposed control task specifications) can be chosen. More details about the procedure can be found in [5], 8]. The graphical tool GraSim was developed for DEDS modelling, analyzing and the control synthesis. The input of the tool is the RG of the PN model. It is created by means of clicking (by the mouse) on icons representing the RG nodes and edges as well as the marks for designating the initial and terminal states. On its output the tool yields (in the 
graphical form) the system trajectories from a given initial state to a prescribed terminal one. The trajectories can be analyzed one after another.

\section{Conclusions}

The alternative PN-based approach to modelling, analysis and control of the agent interactions in MAS was presented in this paper. It yields the results in analytical terms as well as graphically. The PN-based model of the interaction proces is created in analytical terms, the RG and the space of reachable states are generated. Feasible state trajectories are found by means of the mutual intersection of both the SLRT and BTRT. The graphical tool GraSim was developed to automate the control synthesis.

\section{References}

1. Brams, S.J., Kilgour, D.M.: Fallback bargaining. Group Decision and Negotiation 10 (2001) 287-316

2. Čapkovič, F.: Modelling and Control of Discrete Event Dynamic Systems. BRICS Report Series, RS-00-26, University of Aarhus, Denmark (2000) 58 p.

3. Čapkovič, F.: Control synthesis of a class of DEDS. Kybernetes. The Int. Journ. of Sys. and Cybern. 31 No 9/10 (2002) 1274-1281

4. Čapkovič, F.: The generalised method for solving problems of DEDS control synthesis. Lecture Notes in Artificial Intelligence 2718 (2003) 702-711

5. Capkovič, F.: An application of the DEDS control synthesis method. Lecture Notes in Computer Science 3038 Part III (2004a) 528-536

6. Čapkovič, F.: DEDS control synthesis problem solving. In: Proc. 2nd IEEE Conf. on Intelligent Systems, Varna, Bulgaria. IEEE Press (2004b) 299-304

7. Čapkovič, F., Čapkovič, P.: Petri net-based automated control synthesis for a class of DEDS. In: Proc. 2003 IEEE Conf. on Emerging Technologies and Factory Automation. Lisbon, Portugal. IEEE Press (2003) 297-304

8. Capkovič, F.: An application of the DEDS control synthesis method. Journal of Universal Computer Science 11 No 2 (2005) 303-326

9. Hung, P.C.K., Mao, J.Y.: Modeling e-negotiation activities with Petri nets. In: Proc. 35th Hawaii Int. Conf. on System Sciences. Big Island, Hawaii, Vol. 1. IEEE Computer Society Press (CD/ROM) (2002) 10 p.

10. Looney, C.G.: Fuzzy Petri nets for rule-based decision-making. IEEE Trans. Syst. Man Cybern. 18 No 1 (1988) 178-183

11. Nowostawski, M., Purvis, M., Cranefield, S.: A layered approach for modelling agent conversations. In: Proc. 2nd Int. Workshop on Infrastructure for Agents, MAS, and Scalable MAS, Montreal, Canada (2001) 163-170.

12. Purvis, M., Cranefield, S., Nowostawski, M., Ward, R., Carter, D., Oliveira, M.A.: Agentcities interaction using the opal platform. In: Proc. Workshop on Agentcities: Research in Large-Scale Open Agents Environments, 1st Int. Joint Conf. on Autonomous Agents and Multi-Agent. Bologna, Italy. CD/ROM. (2002) 5 p.

13. Thompson, L.: The Mind and Heart of the Negotiator. Prentice-Hall Inc. (1998)

14. Yen, J., Yin, J., Ioerger, T.R., Miller, M.S., Xu, E., Volz, R.A.: Cast: Collaborative agents for simulating teamwork. In: Proc. 17th Int. Joint Conf. on Artificial Intelligence - IJCAI'2001, Seatle, USA (2001) 1135-1142. 